\title{
A Rigorous Proof on the Crystallographic Restriction Theorem to Establish Human Being
}

\author{
Zhang Yue \\ Department of Physics, Hunan Normal University, Changsha, People's Republic of China, 410081 \\ physzhangyue@126.com
}

\begin{abstract}
It is significant to find a more rigorous and satisfactory proof of the crystallographic restriction theorem. The inexistence of $C_{5}$ axis of symmetry is equivalent of that pentagons are impossible to fill all the space with a connected array of pentagons, on the basis of this viewpoint, using a purely mathematical approach the paper rigorously proves that $C_{5}$ and $C_{n}$ $(n \geq 7)$ axes of symmetry cannot exist, and one -, two -, three -, four - and six - fold axes of rotational symmetry are allowable. Therefore, the axes of symmetry of the crystal can merely exist $C_{1}, C_{2}, C_{3}, C_{4}$ and $C_{6}$.
\end{abstract}

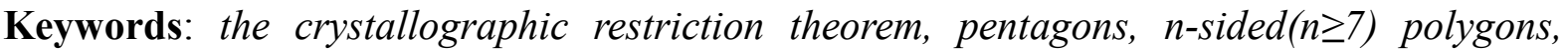
proper rotation.

PACS : 61.50. Ah ; 02.40. Dr

关于晶体制约定理的一个严格证明

张跃

(湖南师范大学物理系, 中国长沙, 410081)

[摘要] 关于晶体制约定理, 有必要研究和提出更加严格和完美的证明。不存在 $\mathrm{C}_{5}$ 轴等 价于不能够用相互之间无任何空隙的五边形填充满所有的空间，以这一观点为基础， 本文利用纯粹的数学方法严格地证明了不存在晶体的 $\mathrm{C}_{5}$ 和 $\mathrm{C}_{\mathrm{n}}(\mathrm{n} \geq 7$ )对称轴, 而允许存 在 $1,2,3,4$ 以及 6 重转动对称轴, 从而证明了晶体的转动对称轴只能够存在 $\mathrm{C}_{1}$, $\mathrm{C}_{2}, \mathrm{C}_{3}, \mathrm{C}_{4}$ 和 $\mathrm{C}_{6}$ 。

关键词: 晶体制约定理, 五边形, $n(n \geq 7)$ 多边形, 固有转动.

\section{Introduction}

It is well - known that the axes of symmetry of the crystal can merely exist $\mathrm{C}_{1}, \mathrm{C}_{2}, \mathrm{C}_{3}$, $\mathrm{C}_{4}$ and $\mathrm{C}_{6}$, this is the so - called crystallographic restriction theorem ${ }^{[1-4]}$. Among various proofs of this theorem, there is a famous proof which is generally concurred by those people who are familiar with the solid state physics. Although the well - known proof of the theorem has been applied in many famous books ${ }^{[2-3]}$, it is not satisfactory at least due to the following two reasons. First, considering an $\mathrm{n}$-fold ( $\mathrm{n}$ is an integer) rotation of the crystal in the two dimensional space, as shown as Fig . $1^{[2]}$,

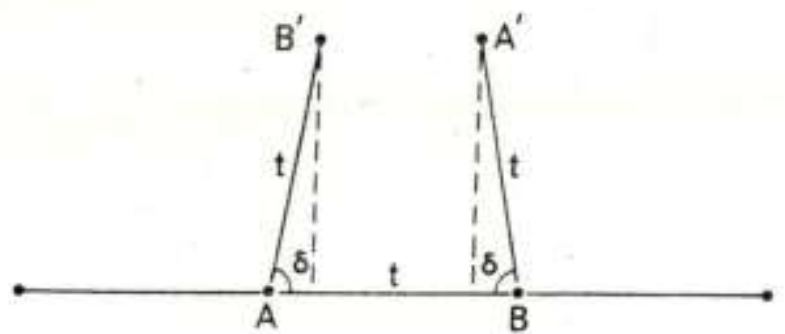

Fig. 1, The point B' is one of the points generated by an $n$-fold rotation axis through point A 
operating on lattice point B with angle $\delta$, and the point A' by a similar axis through point $\mathrm{B}$

inversely operating on lattice point A with angle $\delta$. The value of angle $\delta$ is equal to $\frac{2 \pi}{n}$.

图 $1 \mathrm{~B}^{\prime}$ 点是格点 $\mathrm{B}$ 绕通过格点 $\mathrm{A}$ 处的一个 $\mathrm{n}$ - 重转动轴转动 $\delta$ 角度产生的一个点, $\mathrm{A}^{\prime}$ 点 是格点 $\mathrm{A}$ 绕通过格点 $\mathrm{B}$ 处的一个类似的轴向相反的方向转动 $\delta$ 角度产生的一个点。 $\delta$ 角 的大小为 $\frac{2 \pi}{n}$ 。

in terms of the proof, because of the periodicity of lattice structure, the length of B'A' must be equal to the integral multiples of that of $A B$, namely,

$$
\mathrm{B}^{\prime} \mathrm{A}^{\prime}=\mathrm{mt} \text {. }
$$

However, in the viewpoint of mathematics, eq. (1) is not clear as an argument for the 14 different Bravais lattice structures of real crystals , but not the supposing Bravais lattice, all of the 14 lattices should be respectively demonstrated in order to support eq. (1).

Second, the calculation from eq. (1) in accordance with Fig .1 demonstrates that the possible values of $m$ are $-1,0,1,2$, and 3 , nevertheless, if $m$ takes the value of -1 , neither of the lengths of B'A' and $t$ in eq. (1) can be significant to be negative . theorem .

Therefore, it is necessary to find a rigorous proof of the crystallographic restriction

\section{Review of Literature}

The inexistence of $\mathrm{C}_{5}$ axis is equivalent of that pentagons are impossible to fill all the space with a connected array of pentagons ${ }^{[3]}$, and this can be easily generalized to all the cases of $C_{n}(n \geq 7)$ axis . At first, let us consider two congruent regular pentagons such as $A_{5}$ and $B_{5}$ in the two - dimensional space, as shown as Fig.2 .

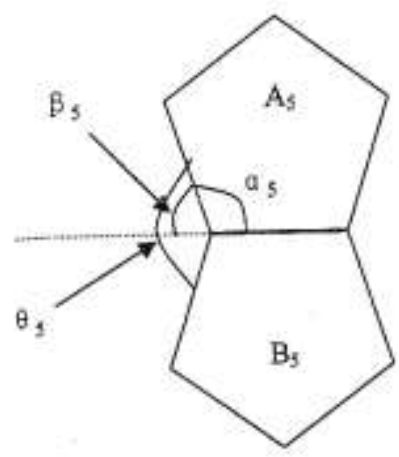

Fig. 2, Two congruent regular pentagons fit together .

图 2 两个全等的正五边形拼接在一起。

In Fig. $2, \alpha_{5}$ and $\beta_{5}$ respectively note the interior angle and the exterior angle of the pentagon, and $\theta_{5}$ is the clipped angle between the side of $A_{5}$ and the side of $B_{5}$. Because the sum of all the exterior angles of a polygon is always equal to $360^{\circ}$, thus, it can be written

$$
\beta_{5}=\frac{360^{\circ}}{5}=72^{\circ} \text {, }
$$

hence, 


$$
\alpha_{5}=180^{\circ}-\beta_{5}=108^{\circ} .
$$

It is clear from Fig. 2 that if the other one or more pentagons just can fill the space within the scope of $\theta_{5}$ with no "gaps" between pentagons, it requires that one or more interior angles can just fill $\theta_{5}$ angle with no "gaps" between them, or the size of $\theta_{5}$ must be just equal to the integral multiples ( positive ) of the size of an interior angle, namely,

$$
\theta_{5}=\mathrm{m} \alpha_{5}(\mathrm{~m}=1,2,3 \ldots \ldots) .
$$

Nevertheless, using eq. (2), the size of $\theta_{5}$ is given by

$$
\theta_{5}=2 \beta \beta_{5}=2 \times 72^{\circ}=144^{\circ} \text {, }
$$

from eq. (5) and eq. (3), it can be found

$$
\theta_{5}=\frac{4}{3} \alpha_{5},
$$

in terms of eq. (4), $\mathrm{C}_{5}$ axis can not exist . Fig .3 depicts the "gaps" between pentagons in the scope of $\theta_{5}$ in a close packing of pentagons in the two-dimensional space ${ }^{[3]}$.

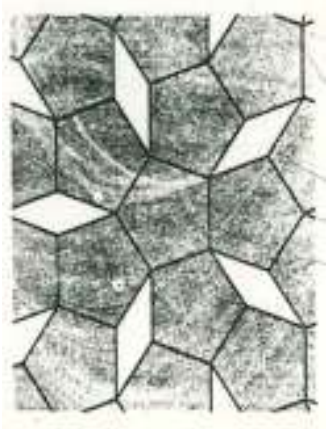

Fiq .3, $\mathrm{C}_{5}$ axis of symmetry does not exist .

图 $3 \mathrm{C}_{5}$ 对称轴不存在。

\section{Discussion}

Assuming to substitute two congruent regular $\mathrm{n}-$ sided $(\mathrm{n} \geq 7)$ polygons such as $\mathrm{A}_{\mathrm{n}}$ and $B_{n}$ respectively for $A_{5}$ and $B_{5}$ in Fig . 2, accordingly, $\theta_{n}\left(\right.$ or $\left.\beta_{n}\right)$ for $\theta_{5}\left(\right.$ or $\left.\beta_{5}\right)$, and $\alpha_{n}$ for $\alpha_{5}$. Thus, an exterior and an interior angles of the $n-\operatorname{sided}(n \geq 7)$ polygon are respectively written

$$
\beta_{\mathrm{n}}=\frac{360^{\circ}}{\mathrm{n}}=\frac{2}{\mathrm{n}} \times 180^{\circ},
$$

and

$$
\alpha_{\mathrm{n}}=180^{\circ}-\beta_{\mathrm{n}}=180^{\circ}-\frac{360^{\circ}}{\mathrm{n}}=\frac{\mathrm{n}-2}{\mathrm{n}} \times 180^{\circ},
$$

in accord with Fig. 2,

$$
\theta_{\mathrm{n}}=2 \beta_{\mathrm{n}}=2 \times \frac{360^{\circ}}{\mathrm{n}}=\frac{4}{\mathrm{n}} \times 180^{\circ} .
$$

Comparing eq. (9) with eq. (8), obviously, if $n \geq 7$, the inequality

$$
\theta_{\mathrm{n}}<\alpha_{\mathrm{n}}
$$

holds true, thus

$$
\theta_{\mathrm{n}} \neq \mathrm{m} \alpha_{\mathrm{n}}(\mathrm{m}=1,2,3 \ldots \ldots),
$$

therefore, $C_{n}(n \geq 7)$ axis can not exist . 
In the viewpoint of mathematics, $\mathrm{C}_{1}, \mathrm{C}_{2}, \mathrm{C}_{3}, \mathrm{C}_{4}$ and $\mathrm{C}_{6}$ axes must also be discussed . At first, it is clear that $\mathrm{C}_{1}$ axis represents an one-fold rotation with the rotation angle $0^{\circ}$ or $360^{\circ}$, and will certainly remain the crystal invariant .

We separately consider an oblique Bravias lattice in the two-dimensional space, if a two-fold rotation with the rotation angle of $180^{\circ}$ through any lattice point in a primitive cell, the primitive cell will remain invariant, this is also true for equivalent points in other primitive cells ${ }^{[3]}$. Therefore, $\mathrm{C}_{2}$ axis for a crystal based on such a primitive cell can exist .

Differing from the case of pentagons, in Fig. 2, If assuming respectively to substitute two congruent regular triangles such as $\mathrm{A}_{3}$ and $\mathrm{B}_{3}$, tetragons such as $\mathrm{A}_{4}$ and $\mathrm{B}_{4}$ and hexagons such as $A_{6}$ and $B_{6}$ for $A_{5}$ and $B_{5}$, accordingly, $\theta_{k}\left(\right.$ or $\left.\beta_{k}\right)(k=3,4,6)$ for $\theta_{5}$ (or $\left.\beta_{5}\right)$, and $\alpha_{k}$ $(\mathrm{k}=3,4,6)$ for $\alpha_{5}$, it is easy to calculate out :

$$
\theta_{3}=4 \alpha_{3}, \theta_{4}=2 \alpha_{4}, \theta_{6}=\alpha_{6},
$$

In accordance with eq. (4), it can be recognized that $\mathrm{C}_{3}, \mathrm{C}_{4}$, and $\mathrm{C}_{6}$ axes are allowable for the crystal rotation .

\section{Conclusion}

With respect to the crystallographic restriction theorem, the paper proposed different opinions on a proof applied in many famous books. Due to the periodicity of the lattice structure, the inexistence of $\mathrm{C}_{5}$ axis is equivalent of that pentagons are impossible to fill all the space with a connected array of pentagons, for example, in Fig.2, if one or more other congruent regular pentagons can fill all the space within the scope of $\theta_{5}$ with no "gaps" between them, the value of $\theta_{5}$ must be the integral multiples of the value of an interior angle of the pentagon. Nevertheless, from the calculation it can be found that the value of $\theta_{5}$ is not an integral multiple of that of $\alpha_{5}$, therefore, $C_{5}$ axis do not exist.

Similarly, if assuming to substitute two congruent regular $n$-sided ( $\mathrm{n} \geq 7$ ) polygons for the two pentagons in Fig.2, the present calculation demonstrates that the value of $\theta_{n}(n \geq 7)$ is smaller than that of the interior angle of the $n$-sided $(n \geq 7)$ polygon, no possible to be its integral multiple. But differing from these cases, if assuming to substitute the two pentagons in Fig . 2 with two congruent regular triangles, or tetragons, or hexagons, it is easy to calculate out that $\theta_{3}=4 \alpha_{3}, \theta_{4}=2 \alpha_{4}, \theta_{6}=\alpha_{6}$, they are consistent with eq. (4). Moreover, it is clear that $C_{1}$ and $\mathrm{C}_{2}$ are compatible with translational symmetry. Therefore, the possible axes of rotation of the crystal are merely $\mathrm{C}_{1}, \mathrm{C}_{2}, \mathrm{C}_{3}, \mathrm{C}_{4}$ and $\mathrm{C}_{6}$.

\section{References}

[1] Sharma B D. Restrictions upon Rotation and Inversion Axes in Crystals [J]. J Chem Educ, 1983, 60 (6): 462 - 463.

[2] Animalu A O E. Intermediate Quantum Theory of Crystalline Solids [M]. Englewood Cliffs: Prentice - Hall, Inc., 1977.

[3] Kittel C. Introduction to Solid State Physics (Sixth Edition) [M]. New York : John Wiley \& Sons, Inc., 1986.

[4] Hiller H. The crystallographic restriction in higher dimensions [J]. ACTA CRYST A, 1985, 41(6) :541 - 544. 